\title{
Suppression of antibiotic resistance evolution by single-gene deletion
}

Takaaki Horinouchi ${ }^{1}$, Tomoya Maeda $\mathbb{1}^{1}{ }^{1}$, Hazuki Kotani ${ }^{1} \&$ Chikara Furusawa ${ }^{1,2 *}$

Antibiotic treatment generally results in the selection of resistant bacterial strains, and the dynamics of resistance evolution is dependent on complex interactions between cellular components. To better characterize the mechanisms of antibiotic resistance and evaluate its dependence on gene regulatory networks, we performed systematic laboratory evolution of Escherichia coli strains with single-gene deletions of 173 transcription factors under three different antibiotics. This resulted in the identification of several genes whose deletion significantly suppressed resistance evolution, including arcA and gutM. Analysis of double-gene deletion strains suggested that the suppression of resistance evolution caused by arcA and gutM deletion was not caused by epistatic interactions with mutations known to confer drug resistance. These results provide a methodological basis for combinatorial drug treatments that may help to suppress the emergence of resistant pathogens by inhibiting resistance evolution.

The emergence of virtually untreatable multidrug-resistant bacteria is an increasing public health concern worldwide ${ }^{1,2}$. Bacterial cells inevitably evolve resistance when this offers a selective advantage under clinical doses of antibiotics, resulting in ineffective antibiotic treatment ${ }^{3}$. Although newly developed compounds have been introduced to treat such resistant bacteria ${ }^{4}$, further resistance acquisition to these compounds often occurs ${ }^{5}$. Furthermore, the development of new antibiotics has declined recently ${ }^{6}$, hence there is a need to develop novel methodologies to suppress the emergence of antibiotic-resistant bacteria.

One approach to combat drug resistance is the use of multidrug combinations, either sequentially or simultaneously ${ }^{7,8}$. It has been demonstrated that drug pairs with synergistic interactions can suppress the emergence of resistance by reducing the time window available for resistance evolution ${ }^{8}$. Furthermore, drug pairs with antagonistic interactions have been shown to suppress resistance evolution by limiting the range of drug concentrations in which resistance is selected ${ }^{9,10}$. In addition to these synergistic and antagonistic drug interactions, collateral sensitivity ${ }^{11}$, i.e., the phenomenon where resistance evolution to one drug induces sensitivity to another, is expected to play an important role in the development of therapeutic strategies to control resistance evolution ${ }^{12}$. Cycling doses and simultaneous addition of drug pairs displaying collateral sensitivity have been shown to result in significant suppression of resistance evolution, providing valuable information for the design of future drug regimes $^{12,13}$.

Several studies have identified tight networks of drug-drug interactions and collateral resistance/sensitivity among drugs ${ }^{14-16}$, indicating complex interactions between the mechanisms of drug responses and resistance evolution. For example, several studies have demonstrated that the collateral sensitivity between $\beta$-lactam and aminoglycoside observed in Escherichia coli (E. coli) is caused by changes in the proton-motive force (PMF) across the cell membrane and respiratory activity ${ }^{16,17}$. Through these studies it has become clear that resistance evolution involves changes in various cellular functions, such as metabolic activity, membrane transport, transcription, and translation. Thus, to design effective mechanisms to suppress resistance evolution, we need to decipher the complex interactions between resistance evolution and these cellular functions. However, in previous studies, the analyses of such interactions have been limited to responses and resistance acquisition to known antibiotics, resulting in a failure to reveal critical molecular mechanisms affecting the dynamics of resistance evolution.

In this study, to systematically investigate mechanisms to suppress antibiotic resistance evolution, we performed laboratory evolution of single-gene deletion strains ${ }^{18}$ of $E$. coli in the presence of three antibiotics with different targeting mechanisms. We used deletion strains of transcription factors (TFs) as the ancestors of the laboratory evolution, as their deletion is expected to perturb a wide range of cellular functions. We screened for TF genes whose deletion significantly suppressed or accelerated antibiotic resistance evolution. Based on the results, we discuss strategies to develop drug combinations that could inhibit antibiotic resistance evolution thereby improving the success of future antibiotic treatments.

${ }^{1}$ Center for Biosystems Dynamics Research, RIKEN, 6-2-3 Furuedai, Suita, Osaka, 565-0874, Japan. ${ }^{2}$ Universal Biology Institute, The University of Tokyo, 7-3-1 Hongo, Tokyo, 113-0033, Japan. *email: chikara.furusawa@riken.jp 
(a)

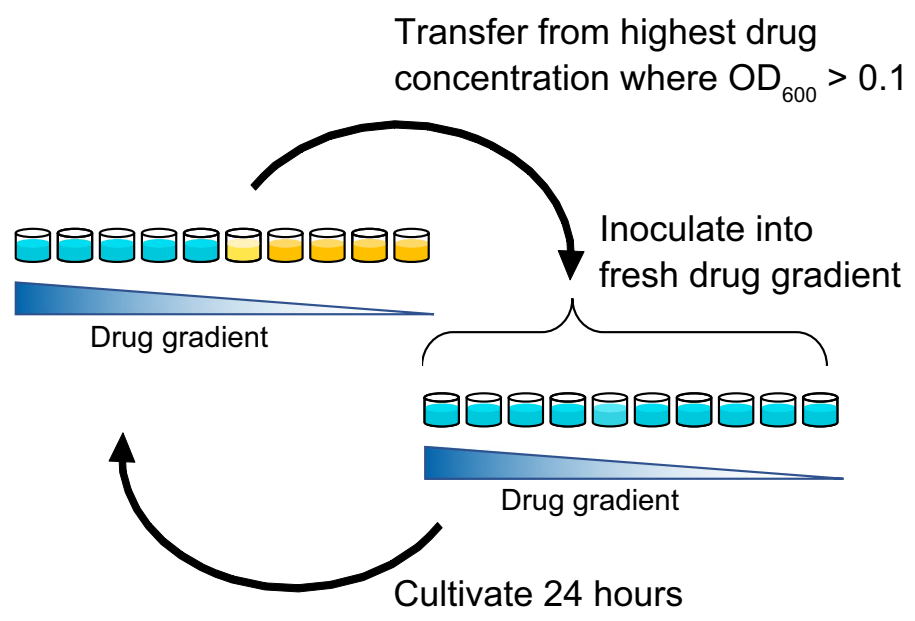

(b)

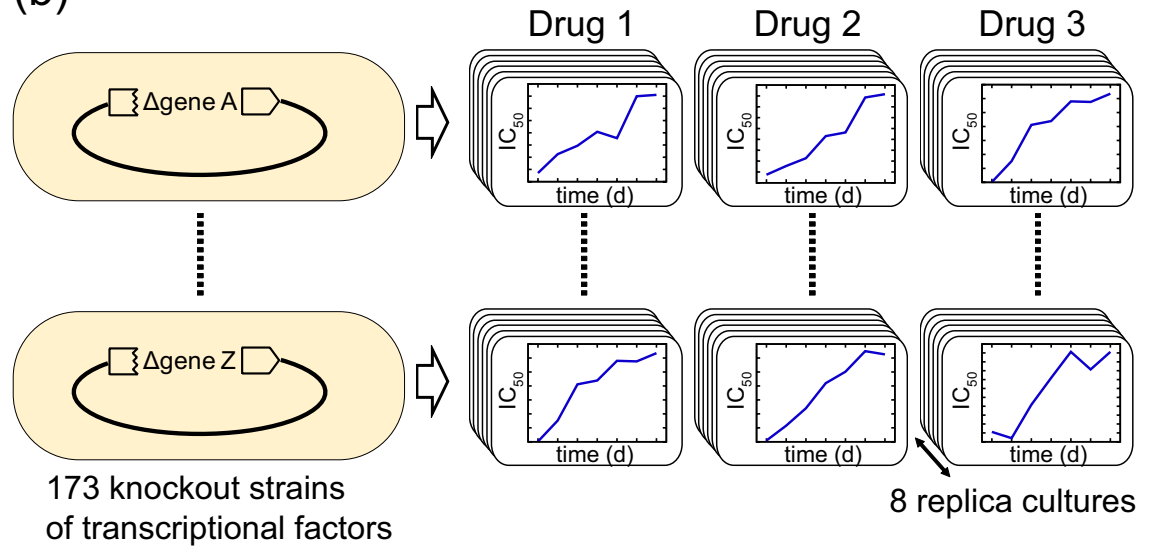

(c)

(d)
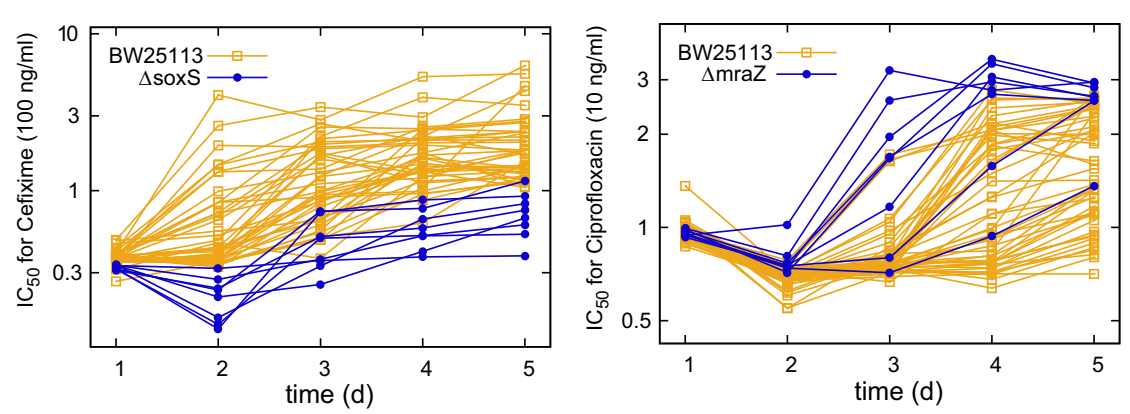

Figure 1. Laboratory evolution using single-gene deletion strains as ancestors. (a) The method of laboratory evolution by serial dilution. Every 24 hours, cells from the well with the highest drug concentration that exhibited an $\mathrm{OD}_{620}>0.1$ were transferred to fresh medium with the drug gradient. (b) Design of the experiment. We used 173 deletion strains of transcription factors as the ancestor strains for laboratory evolution in three different antibiotics. (c,d) Examples of the time courses of $\mathrm{IC}_{50}$ during laboratory evolution. $\mathrm{Th}^{\mathrm{IC}} \mathrm{IC}_{50}$ time courses of (c) the $\Delta$ soxS strain under Cefixime and (d) the $\Delta m r a Z$ strain under Ciprofloxacin (blue lines) are presented with those of the wild-type strain BW25113 (without gene deletion - yellow lines). The time courses of 8 and 40 replicates are shown for the deletion strains and BW25113, respectively.

\section{Results}

Laboratory evolution of single-gene deletion strains under antibiotics. Figure 1 shows a schematic of the experimental design of this study. To investigate the effect of gene deletion on antibiotic resistance evolution, we evolved E. coli strains obtained from the Keio single-gene deletion library ${ }^{18}$ in the presence of 3 antibiotics. The drugs cover three major antibiotic targets in E. coli: cell wall synthesis (Cefixime; CFIX), DNA replication (Ciprofloxacin; CPFX), and protein synthesis (Chloramphenicol; CP). We selected 173 deletion strains of TFs from the deletion library as ancestors of the laboratory evolution (the list of deleted genes is shown in Table S1). 
The laboratory evolution experiments were carried out using $2^{0.5}$ (CFIX and CPFX) or $2^{0.25}$ (CP)-fold dilution gradients in 384-well plates. Cells were propagated daily from the well containing the highest drug concentration that exceeded a given threshold of an optical density at $620 \mathrm{~nm}\left(\mathrm{OD}_{620}\right)$ (Fig. 1a; see Materials and methods for details). To evaluate the reproducibility of the evolutionary dynamics, 8 independent culture lines were propagated in parallel for each antibiotic/ancestor combination. The wild-type strain BW25113, which is the host strain of the gene deletion library, was used as a control. More than 4,000 independent culture series were maintained (173 deletion strains $\times 3$ drugs $\times 8$ replicates and controls; Fig. 1b). The evolution was performed for 5 passages (CFIX and CPFX) or 8 passages (CP), resulting in significant increases in drug resistance in the wild-type strain BW25113 (without gene deletion). All experiments were performed by an automated culture system that we previously developed for laboratory evolution ${ }^{19}$.

To quantify drug resistance, we calculated $50 \%$ inhibition levels $\left(\mathrm{IC}_{50}\right.$ ) from the $\mathrm{OD}_{620}$ measurements in the daily propagation. Figures $1 \mathrm{c}$,d show examples of the time course of $\mathrm{IC}_{50}$ during laboratory evolution. The yellow lines represent the resistance evolution of BW25113 (without gene deletion, $n=40$ ), while the blue lines correspond to the resistance evolution of $\Delta$ soxS under CFIX (Fig. 1c) and $\Delta m r a Z$ under CPFX (Fig. 1d) $(\mathrm{n}=8)$. The deletion of soxS, encoding the activator of the superoxide response regulon ${ }^{20}$, suppressed resistance evolution to the cell wall synthesis inhibitor CFIX, whereas the deletion of $m r a Z$, encoding a repressor that controls cell division and cell wall synthesis ${ }^{21}$, accelerated resistance evolution to the DNA replication inhibitor CPFX. All plots for the 173 deletion strains are shown in Figs. S1-S3 and the $\mathrm{IC}_{50}$ values are presented in Table S2.

Impact of gene deletion on resistance evolution. Figure 2 shows the relationship between drug resistance before and after evolution, where each dot represents the mean $\mathrm{IC}_{50}$ of the 8 replica culture series for the 173 deletion strains. The data for the wild-type strain BW25113 (without deletion) are also plotted. The $x$-axis and $y$-axis show the $\mathrm{IC}_{50}$ values calculated from the dose-response curves on the first day and the last day, respectively. Most data points are located above the dotted reference line $(y=x)$, indicating an increase in drug resistance during the laboratory evolution.

In Fig. 2, the variance of $\mathrm{IC}_{50}$ values on the $x$-axis represents the effect of deleting TFs on the drug resistance. For example, the deletion of $o m p R$ exhibited a significant increase in $\mathrm{IC}_{50}$ to all three drugs. It is known that the deletion of $\operatorname{ompR}$ causes decreased expression of $\mathrm{OmpF}$ outer membrane porins ${ }^{22}$, which leads to resistance to various drugs. In contrast, the deletion of $l y s R$ causes sensitivity to all three drugs we investigated.

The $\mathrm{IC}_{50}$ values on the $y$-axis provide information on how the deletion of TFs affects the resistance evolution. For example, before laboratory evolution, the deletion strain of $r p o S$, encoding sigma factor $\sigma^{\mathrm{s}}$ of RNA polymerase ${ }^{23}$, exhibited a similar $\mathrm{IC}_{50}$ to CFIX as the wild-type strain BW25113, and there was little increase of $\mathrm{IC}_{50}$ during the laboratory evolution (depicted by an arrow in Fig. 2a). On the other hand, the deletion strain of $r p o S$ acquired resistance to CPFX and $\mathrm{CP}$ by laboratory evolution (Fig. S4), which may suggest that $r p o S$-mediated regulation plays a more important role in the resistance evolution to CFIX than the other two drugs. The advantage of systematic laboratory evolution is that we can distinguish the effect of gene deletion on drug resistance from its impact on resistance evolution.

One possible explanation for the changes in $\mathrm{IC}_{50}$ shown in Fig. 2 is changing growth rate caused by deletion of TFs. To check this possibility, we quantified the specific growth rate and the cell concentration at the stationary phase for the TF deletion strains, and analyzed the relationship to the $\mathrm{IC}_{50}$ values after evolution under antibiotics. As shown in Fig. S5, there was no correlation between the change in $\mathrm{IC}_{50}$ and the growth rate/final cell concentration in the TF deletion strains. This result suggests that the change of $\mathrm{IC}_{50}$ shown in Fig. 2 is not caused by changes in the growth rate. Another possible explanation of the changes in resistance evolution in Fig. 2 is the changing mutation rate by the TF deletions. To check this possibility, we compared the change in mutation rate of $12 \mathrm{TF}$ deletion strains obtained by previous study ${ }^{24}$ and $\mathrm{IC}_{50}$ values on the last day, as shown in Fig. S6. The results demonstrated that there was no correlation between the change in the mutation rate and the observed changes in the resistance evolution.

Genes whose deletion suppresses antibiotic resistance evolution. Based on the time series data of $\mathrm{IC}_{50}$ during the laboratory evolution, we screened genes whose deletion significantly suppressed resistance evolution. For the screening, we used the Wilcoxon rank sum test between $\mathrm{IC}_{50}$ values of a deletion strain and the wild-type BW25113 strain on the last day of laboratory evolution. The Venn diagram in Fig. 3a shows the 42 genes whose deletion strains exhibited significantly lower $\mathrm{IC}_{50}$ values than the control strain (false discovery rate (FDR) is less than 0.1). The Venn diagram for the acceleration of resistance evolution, containing a total of 21 genes, is shown in Fig. S7. As can be seen in Fig. 3a, the deletion strains of four genes (lysR, arcA, gutM, fur) had significantly lower $\mathrm{IC}_{50}$ values for all three drugs. Of these, the $\Delta l y s R$ and $\Delta$ fur strains also exhibited significantly lower $\mathrm{IC}_{50}$ than other strains on the first day, as demonstrated that they can be statistically excluded at outliers $(p<0.01$; chi-squared test for outliers) in the case of CP resistance. Here, we exclude $\Delta l y s R$ and $\Delta f u r$ from the following analysis, since for these strains, the resistance acquisition during laboratory evolution was difficult to be evaluated by the $\mathrm{IC}_{50}$ values on the last day. In this analysis, many deletion strains exhibited significantly different resistance levels on the first day from the wild-type strain (Table S2). However, the absolute differences in $\mathrm{IC}_{50}$ values are not always large, and in this study, we focus on the genes whose deletion strains showed similar $\mathrm{IC}_{50}$ values on the first day, and different $\mathrm{IC}_{50}$ values after evolution, as $\Delta \operatorname{arc} A$ and $\Delta g u t M$ strains. Figures $3 \mathrm{~b}-\mathrm{g}$ show the time course of $\mathrm{IC}_{50}$ during the adaptive evolution of $\triangle a r c A$ and $\triangle g u t M$, respectively. These deletion strains exhibited similar $\mathrm{IC}_{50}$ values as BW25113 and other deletion strains before the adaptive evolution, while the resistance acquisitions were significantly suppressed.

Analysis of epistatic interactions. As shown in Fig. 3b-g, the deletion of $\operatorname{arcA}$ and gutM suppressed resistance evolution to the three drugs CFIX, CPFX, and CP. One possible explanation for this is that these deletions have negative epistatic interactions with other mutations that contribute to the resistance acquisition to 
(a)

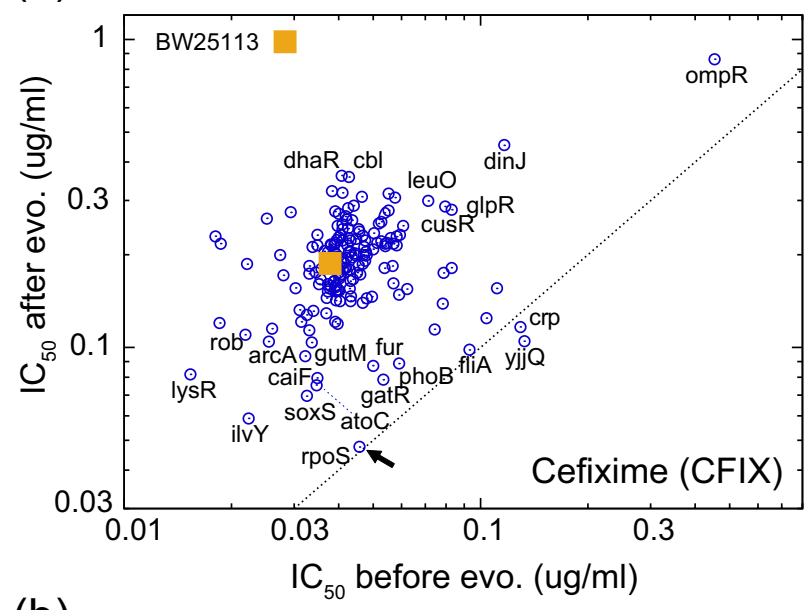

(b)

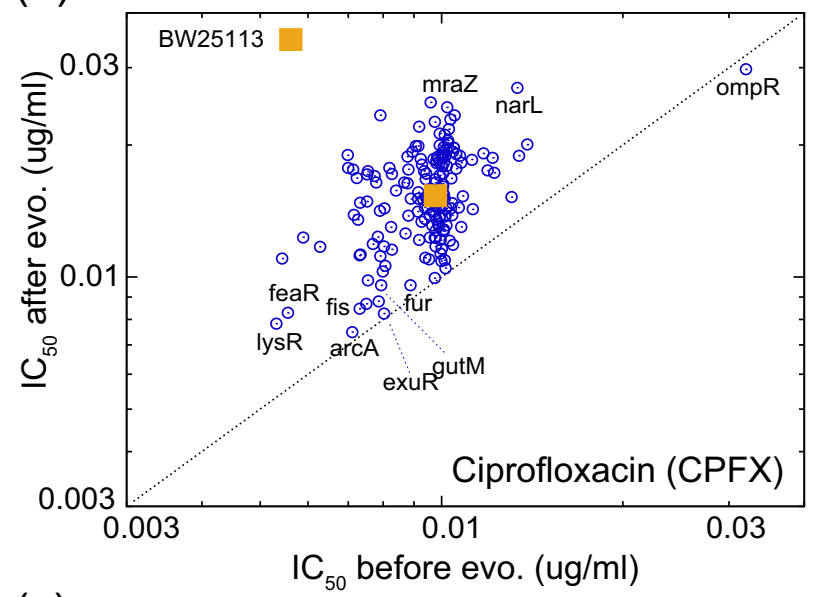

(c)

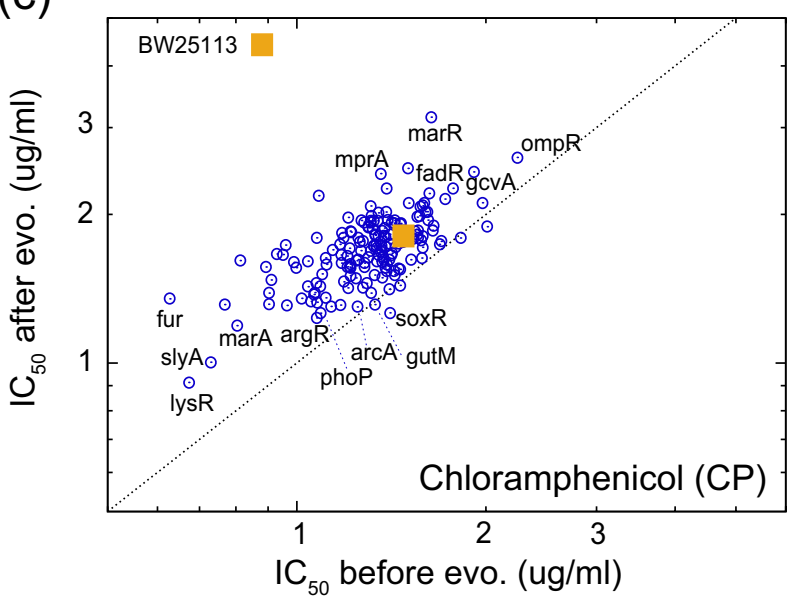

Figure 2. The relationship between $\mathrm{IC}_{50}$ values before and after laboratory evolution. Each dot represents the mean $\mathrm{IC}_{50}$ of 173 deletion strains over 8 replica culture series for (a) CFIX, (b) CPFX, and (c) CP. The average $\mathrm{IC}_{50}$ of the wild-type strain BW25113 (without deletion) for each drug is also presented as the yellow square. Names of some representative genes whose deletion strains show large deviations from the BW25113 control are presented proximal to the corresponding data points. The dotted line shows the function $y=x$ for reference.

these drugs. To check this possibility, we added further genetic alterations to the $\triangle \operatorname{arc} A$ and $\Delta g u t M$ strains. We selected the genes $\operatorname{mpF}$ and $a c r R$ for deletion, as these are known as major contributors to the resistance acquisition to these drugs. Our previous laboratory evolution study showed that deletion strains conferring resistance to these drugs were commonly found to have mutations in these two genes ${ }^{16}$.

Figure 4 shows the $\mathrm{IC}_{50}$ values of the single and double mutant strains for the three drugs. Although the single mutant strains did not exhibit significant increases in CP resistance, the deletion of ompF significantly increased 


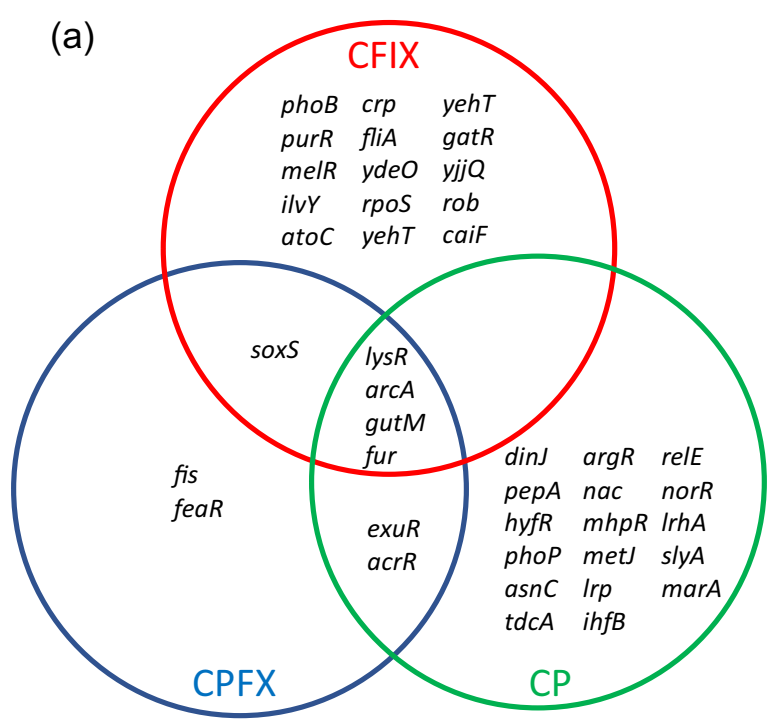

(b)

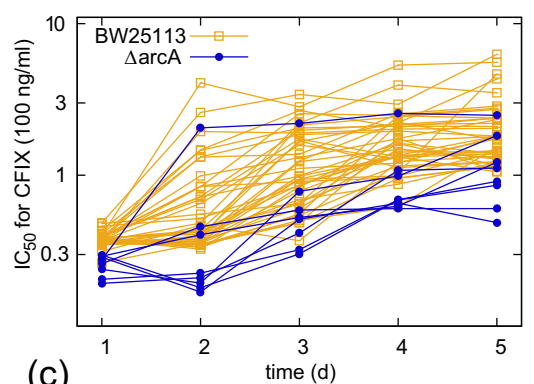

(c)

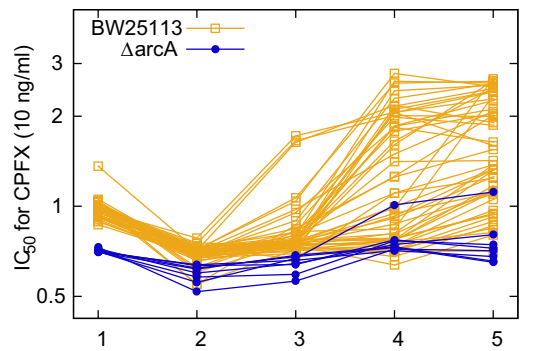

(d)

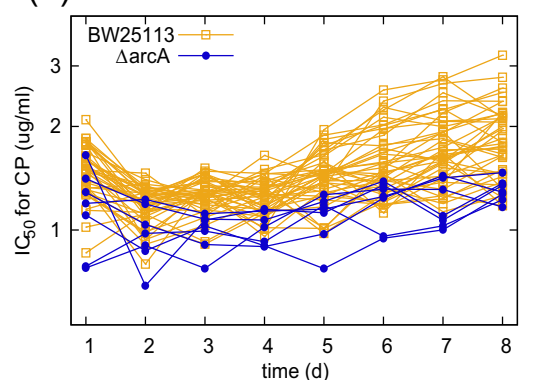

(e)

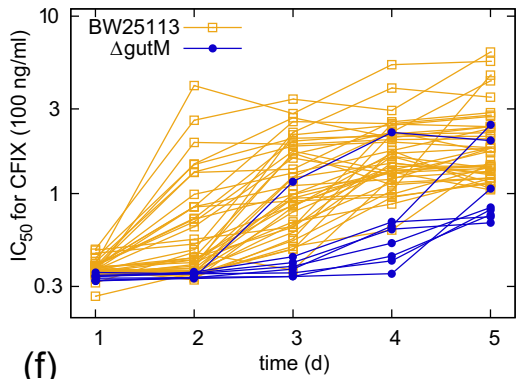

(f)

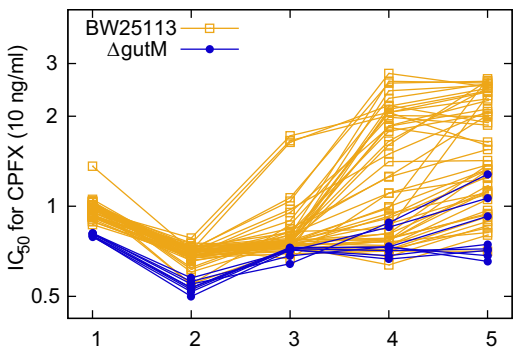

(g)

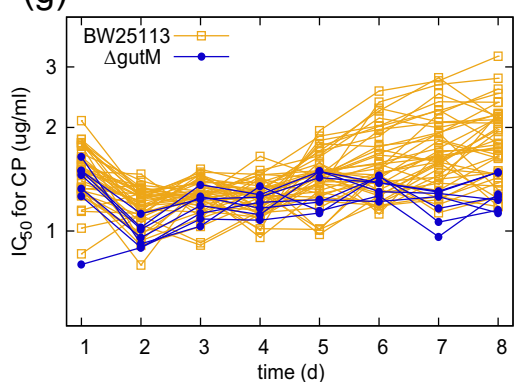

Figure 3. Genes whose deletion significantly suppresses resistance evolution. (a) Venn diagram representing genes whose deletion resulted in significantly lower $\mathrm{IC}_{50}$ values for the indicated drugs (Cefixime; CFIX, Ciprofloxacin; CPFX, Chloramphenicol; $\mathrm{CP}$ ) on the last day of laboratory evolution in comparison with the wild-type strain BW25113 (without gene deletion). The genes with FDR (calculated by Benjamini-Hochberg method) $<0.1$ are presented. $(\mathbf{b}-\mathbf{g})$ The time courses of $\mathrm{IC}_{50}$ for $\triangle \operatorname{arc} A(\mathbf{b}-\mathbf{g})$ and $\triangle$ gut $M(\mathbf{e}-\mathbf{g})$ in the laboratory evolution with the three indicated drugs.

the resistance to CFIX and CPFX, and the deletion of acrR slightly increased the resistance to CPFX. Importantly, in the cases where the additional mutations led to increased resistance, the double deletion strains with $\triangle \operatorname{arc} A$ and $\triangle$ gutM exhibited similar $\mathrm{IC}_{50}$ values to the single deletion strains of $\operatorname{ompF}$ and $a c r R$. This suggests that there is little epistatic interaction between $\operatorname{arcA} / g u t M$ deletions and the $o m p F$ and $a c r R$ mutations which are known to 
(a)

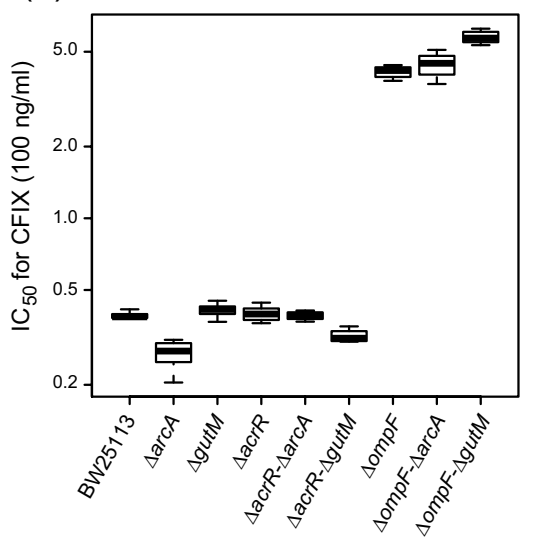

(b)

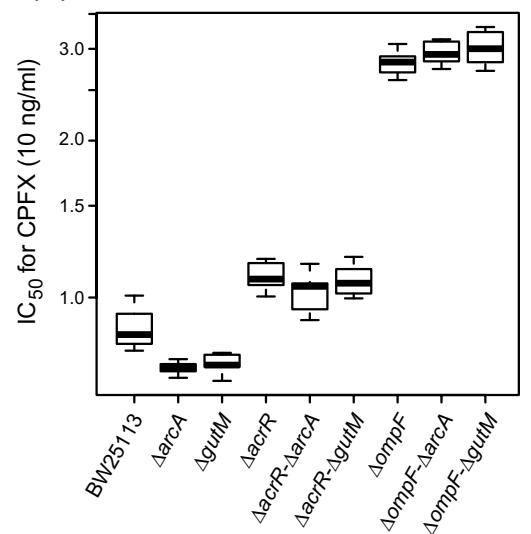

(c)

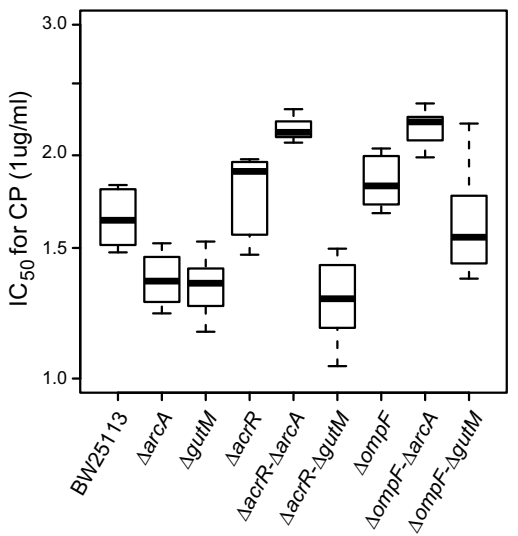

Figure 4. The $\mathrm{IC}_{50}$ values of single and double deletion strains. The boxplots represent $\mathrm{IC}_{50}$ values of 8 replicates for each strain. The IC $_{50}$ values of 9 strains for (a) Cefixime (CFIX), (b) Ciprofloxacin (CPFX), and (c) Chloramphenicol (CP) are presented.

contribute to resistance to CFIX and CPFX. Of course, the possibility remains that other mutations beneficial to drug resistance have negative epistatic interactions with the deletion of $\operatorname{arcA} / g u t M$. However, these results indicate that at least some genes whose mutation is beneficial for drug resistance are present in $\triangle \operatorname{arc} A$ and $\Delta g u t M$ strains, even though they were not mutated during the laboratory evolution. In contrast, for the resistance acquisition to CP, the deletion of gutM did appear to exhibit negative epistatic interactions with the deletion of ompF and $a c r R$, i.e., the double deletion strains $\Delta a c r R-\Delta g u t M$ and $\Delta o m p F-\Delta g u t M$ showed lower $\mathrm{IC}_{50}$ values than the single deletion strains $\triangle a c r R$ and $\Delta o m p F$ (Fig. 4c). This suggests that the observed suppression of resistance acquisition to CP in the deletion strain of gutM was caused by the epistatic interaction with acrR and $\operatorname{ompF}$ mutations. It should be noted that, the specific growth rates of $\Delta a c r R-\Delta g u t M$ and $\Delta o m p F-\Delta g u t M$ strains were $0.46 \pm 0.01$ and $0.37 \pm 0.01(1 / \mathrm{h})$, respectively, under the condition without the addition of antibiotics. The latter was significantly smaller than that of the wild-type strain BW25113, which might suggest that the growth deficiency caused the observed epistatic interaction.

\section{Discussion}

During antibiotic resistance evolution, various molecular mechanisms including gene regulation, membrane transport, and metabolic reactions need to be changed in a highly regulated manner to achieve higher fitness. Thus, to design drug treatments that suppress resistance evolution, we need to understand the complex interplay between the molecular mechanisms related to drug resistance. In this study, we screened for TFs whose deletion affects drug-resistance evolution of E. coli using high-throughput laboratory evolution combined with a single-gene deletion library. The results revealed that resistance evolution was significantly accelerated by the deletion of 21 genes and suppressed by the deletion of 42 genes, suggesting a complicated network between gene disruption and the mechanisms underlying resistance evolution.

We found that the $\operatorname{arc} A$ and $g u t M$ deletion strains exhibited significant suppression of resistance evolution to all three drugs tested, suggesting the involvement of these genes in the mechanisms of antibiotic resistance evolution. The $\operatorname{arcA}$ gene encodes the ArcA/B two-component system, which is a global regulator of gene expression under microaerobic and anaerobic growth conditions ${ }^{25}$. The ArcA/B system represses protein expression involved in aerobic respiration, such as those related to the tricarboxylic acid cycle, and activates gene expression involved in microaerobic or fermentative metabolism in response to redox conditions ${ }^{26}$. Thus, the deletion of $\operatorname{arcA}$ causes activation of aerobic respiration even in microaerobic or anaerobic conditions ${ }^{27}$. This metabolic change might contribute to the observed suppression of resistance evolution. The shift to aerobic metabolism caused by $\operatorname{arcA}$ deletion can enhance production of reactive oxygen species (ROS), and various studies have suggested that ROS production is involved in regulating the activation of antibiotic-mediated cell killing ${ }^{28,29}$. Thus, it is logical to postulate that evolution of antibiotic resistance favors anaerobic metabolism with relatively low ROS production. Our previous study ${ }^{16}$ demonstrated that the expression level of $\operatorname{arcA}$ was significantly increased in strains resistant to $\beta$-lactams, quinolones, and chloramphenicol obtained by laboratory evolution (Fig. S8), supporting the notion of a shift towards anaerobic metabolism. Furthermore, we quantified the intracellular ROS level by using 5-(and-6)carboxy-2', $7^{\prime}$-dichlorodihydrofluorescein diacetate. The result is presented in Fig. S9, which also supported our hypothesis that the deletion of $\operatorname{arc} A$ results in higher ROS production. The activation of ROS production by $\operatorname{arcA}$ deletion could enhance the ROS-mediated effect of antibiotics, potentially suppressing resistance evolution.

The gutM gene encodes a DNA-binding transcription factor that regulates genes related to glucitol utiliza$\operatorname{tion}^{30}$. So far, studies on the function of gutM have focused primarily on glucitol metabolism, therefore it is unclear how the deletion of this gene contributes to the suppression of resistance evolution. However, one study has suggested that gutM is involved in biofilm formation ${ }^{31}$, suggesting that it may have functions related to stress resistance. Further studies will be required to gain more mechanistic insight into how this gene affects antibiotic resistance evolution. 
It should be noted that there was little epistatic interaction between the deletion of $\operatorname{arcA} / \mathrm{gut} M$ and mutation of the genes $o m p F$ and $a c r R$, which are known to be beneficial for resistance to CFIX and CPFX, suggesting that these deletion strains were unable to activate these beneficial mutations. The mechanism for this suppression of resistance acquisition by $\operatorname{arc} A$ and $g u t M$ deletions therefore remains unclear. One hypothesis is that the $\triangle \operatorname{arc} A$ and $\triangle g u t M$ strains transiently show sensitivity to these drugs when beneficial mutations are fixed, resulting in washing out these mutants. To verify this hypothesis, population dynamics under drug treatment could be performed at single-cell level, using microfluidic devices for example. Another possible explanation for the suppression of resistance evolution is a decreased mutation rate in these deletion strains. However, this seems unlikely for the $\triangle \operatorname{arc} A$ strain, in which activation of the respiration pathway enhances the production of ROS, which is known to enhance mutation events via double-strand breaks.

In conclusion, the results of our systematic laboratory evolution study using single-gene deletion strains provided novel insights into targets for reducing the emergence of drug-resistant pathogens. For example, our results suggest that the combinatorial use of antibiotics and compounds that activate the respiration pathway, such as an ArcA phosphorylation inhibitor, might suppress resistance evolution to antibiotics. Although the use of the latter does not directly kill or inhibit the growth of pathogens, it could be beneficial by controlling resistance evolution to the antibiotic. Of course, the number of gene deletions to be analyzed was limited, and epistatic interactions among them remain largely unclear. However, our high-throughput system for laboratory evolution enabled us to quantitatively analyze the effect of gene perturbations on the resistance evolution to antibiotics, and such systematic analysis will be our future scope.

\section{Materials and Methods}

Strains and culture conditions. The E. coli BW25113 strain and the derivative Keio gene deletion library were obtained from the National BioResource Project (NIG, Japan). We selected strains with deletion of all TFs which regulate more than 2 genes according to RegulonDB ${ }^{32}$ (a list of the 173 selected genes is shown in Table S1). The laboratory evolution experiments were performed in modified M9 minimal medium ${ }^{19}$ with $5 \mathrm{~g} / \mathrm{L}$ glucose as the carbon source. The strains were grown individually in 384-well plates (3680, Corning Inc., NY, USA) in $50 \mu l$ of M9 minimal medium with agitation at 300 rotations $/ \mathrm{min}$ at $34^{\circ} \mathrm{C}$. All cultures were performed using an automated culture system consisting of a Biomek ${ }^{\circledR}$ NX span-8 laboratory automation workstation (Beckman Coulter, Tokyo, JP) in a sterile booth connected to a microplate reader (FilterMax F5; Molecular Devices, CA, USA), a shaker incubator (STX44; Liconic, Mauren, LI), and a microplate hotel (LPX220, Liconic, Mauren, LI). Before starting the laboratory evolution, the deletion strains were grown without the addition of antibiotics for at least more than 50 generations.

Laboratory evolution under antibiotics. Eight independent cultures of each deletion strain were propagated in parallel in each concentration of a serial dilution of antibiotics (at slightly lower than the minimum inhibitory concentration). Laboratory evolution was performed at 12 different concentrations of antibiotics using $2^{0.5}$ (CFIX and CPFX) or $2^{0.25}$ (CP)-fold dilution steps. Antibiotic serial diluted plates were prepared using a Biomek ${ }^{\circledR}$ NX span-8 and Biomek ${ }^{\circledR}$ NX MC (Beckman Coulter, CA, USA). The growth of the cells was monitored daily by measuring the $\mathrm{OD}_{620}$ of each well using a microplate reader (FilterMax F5, Molecular Devices, CA, USA). We defined wells with $\mathrm{OD}_{620}$ greater than 0.1 as viable wells. Cells calculated to yield an initial $\mathrm{OD}_{620}$ of $3 \times 10^{-4}$ (corresponding to approximately $1 \times 10^{3}$ cells per well), were transferred from the viable well with the highest drug concentration to new plates with fresh medium and various concentrations of antibiotics.

Data analysis. To obtain the $\mathrm{IC}_{50}$ values, the $\mathrm{OD}_{620}$ values for the dose-response series were fitted to the following sigmoidal model:

$$
f(x)=\frac{a-c}{1+\exp \left\{b \times\left(\log x-\log \mathrm{IC}_{50}\right)\right\}}+c
$$

where $x$ and $f(x)$ represent the concentration of antibiotics and the observed $\mathrm{OD}_{620}$ values, respectively, and $a$, $b$, and $\mathrm{c}$ are fitting parameters. The fitting was performed by a greedy genetic algorithm with a custom-made $\mathrm{C}$ program. All statistical analyses shown in Fig. 3 were performed in R programming language.

Construction of double-deletion mutants. Using Keio single-gene deletion strains as the parental strain, double-deletion mutants were constructed by multiplexed automated genome engineering (MAGE) using a pORTMAGE-4 vector ${ }^{33}$. The plasmid pORTMAGE-4 was a gift from Csaba Pál (Addgene plasmid \#72679). Sequences of MAGE oligos were created using the MODEST tool ${ }^{34}$. An NheI site containing a TAG stop codon and one base frame-shift mutation was inserted into the ORF region of ompF or $a c r R$ to construct double-deletion mutants following the procedure reported previously ${ }^{33}$. Briefly, the MAGE oligo was electroporated into the parental strain and inserted into the target locus by $\lambda$-Red recombinase on the plasmid pORTMAGE- 4 . The genome modifications were verified by colony PCR and Sanger sequencing of the PCR products. The sequences of oligos and primers used in this study are shown in Table S3.

Reactive oxygen species assay. To assess the cellular reactive oxygen species (ROS), 5-(and-6)-carboxy-2', 7 -dichlorodihydrofluorescein diacetate (carboxy- $\mathrm{H}_{2}$ DCFDA) (C400, Invitrogen Co., CA, USA) was used as a fluorescent indicator of the intra-cellular ROS level. Each overnight culture was added to $200 \mu \mathrm{l}$ of fresh M9 medium in the 96-well glass-bottom plate (265300, Thermo Fisher Scientific Inc., MA, USA) and cultivated at $34^{\circ} \mathrm{C}$. The cells in the exponential growth phase were harvested by centrifugation and washed by pre-warmed PBS. As per the manufacturer's instruction, $\mathrm{H}_{2} \mathrm{O}_{2}(100 \mu \mathrm{M}$ final concentration) was added to one set of control 
cells and incubated at $34^{\circ} \mathrm{C}$ for $30 \mathrm{~min}$. Before centrifugation as the positive control for ROS production. After centrifugation, carboxy- $\mathrm{H}_{2}$ DCFDA was added to one set of samples $(10 \mu \mathrm{M}$ final concentration) and incubated at $34^{\circ} \mathrm{C}$ for $30 \mathrm{~min}$. The other set of samples that were incubated without adding carboxy- $\mathrm{H}_{2} \mathrm{DCFDA}$ were used for the measurement of autofluorescence of the cell. The cells were centrifuged and suspended by PBS to remove the extracellular carboxy- $\mathrm{H}_{2} \mathrm{DCFDA}$. The cell density $\left(\mathrm{OD}_{600}\right)$ and fluorescence $\left(\mathrm{Abs}_{535}\right)$ were measured by using the microplate reader (1420 ARVO, PerkinElmer Inc., MA, USA). Specific fluorescence was calculated as follows.

$$
\text { Specific Fluorescence }=\frac{\mathrm{Abs}_{535}\left(\text { carboxy }-\mathrm{H}_{2} \mathrm{DCFDA}[+]\right)}{\mathrm{OD}_{600}\left(\operatorname{carboxy}-\mathrm{H}_{2} \mathrm{DCFDA}[+]\right)}-\frac{\mathrm{Abs}_{535}\left(\operatorname{carboxy}-\mathrm{H}_{2} \mathrm{DCFDA}[-]\right)}{\mathrm{OD}_{600}\left(\operatorname{carboxy}-\mathrm{H}_{2} \mathrm{DCFDA}[-]\right)}
$$

Received: 4 July 2019; Accepted: 12 February 2020;

Published online: 06 March 2020

\section{References}

1. Levy, S. B. \& Marshall, B. Antibacterial resistance worldwide: causes, challenges and responses. Nat. Med. 10, S122-S129 (2004).

2. Alanis, A. J. Resistance to Antibiotics: Are We in the Post-Antibiotic Era? Arch. Med. Res. 36, 697-705 (2005).

3. Davies, J. \& Davies, D. Origins and evolution of antibiotic resistance. Microbiol. Mol. Biol. Rev. 74, 417-433 (2010).

4. Fischbach, M. A. \& Walsh, C. T. Antibiotics for Emerging Pathogens. Science (80-.). 325, 1089-1093 (2009).

5. Bush, K. et al. Tackling antibiotic resistance. Nat. Rev. Microbiol 9, 894-896 (2011).

6. Norrby, S. R., Nord, C. E. \& Finch, R. Lack of development of new antimicrobial drugs: a potential serious threat to public health. Lancet Infectious Diseases 5, 115-119 (2005).

7. Tamma, P. D., Cosgrove, S. E. \& Maragakis, L. L. Combination therapy for treatment of infections with gram-negative bacteria. Clin. Microbiol. Rev. 25, 450-470 (2012).

8. Cottarel, G. \& Wierzbowski, J. Combination drugs, an emerging option for antibacterial therapy. Trends Biotechnol. 25, 547-55 (2007).

9. Chait, R., Craney, A. \& Kishony, R. Antibiotic interactions that select against resistance. Nature 446, 668-671 (2007).

10. Torella, J. P., Chait, R. \& Kishony, R. Optimal drug synergy in Antimicrobial Treatments. PLoS Comput. Biol. 6, e1000796 (2010).

11. Pál, C., Papp, B. \& Lázár, V. Collateral sensitivity of antibiotic-resistant microbes. Trends Microbiol. 23, 401-7 (2015).

12. Munck, C., Gumpert, H. K., Wallin, A. I., Wang, H. H. \& Sommer, M. O. Prediction of resistance development against drug combinations by collateral responses to component drugs. Sci. Transl. Med. 6, 262ra156 (2014).

13. Yoshida, M. et al. Time-programmable drug dosing allows the manipulation, suppression and reversal of antibiotic drug resistance in vitro. Nat. Commun. 8, 15589 (2017).

14. Lázár, V. et al. Genome-wide analysis captures the determinants of the antibiotic cross-resistance interaction network. Nat. Commun. 5, 4352 (2014).

15. Imamovic, L. \& Sommer, M. O. A. Use of Collateral Sensitivity Networks to Design Drug Cycling Protocols That Avoid Resistance Development. Sci. Transl. Med. 5, 204ra132-204ra132 (2013).

16. Suzuki, S., Horinouchi, T. \& Furusawa, C. Prediction of antibiotic resistance by gene expression profiles. Nat. Commun. 5, 5792 (2014).

17. Lazar, V. et al. Bacterial evolution of antibiotic hypersensitivity. Mol. Syst. Biol. 9, 700-700 (2014).

18. Baba, T. et al. Construction of Escherichia coli K-12 in-frame, single-gene knockout mutants: the Keio collection. Mol. Syst. Biol. 2, 2006.0008 (2006).

19. Horinouchi, T., Minamoto, T., Suzuki, S., Shimizu, H. \& Furusawa, C. Development of an automated culture system for laboratory evolution. J. Lab. Autom. 19, 478-82 (2014).

20. Li, Z. \& Demple, B. SoxS, an activator of superoxide stress genes in Escherichia coli: Purification and interaction with DNA. J. Biol. Chem. (1994).

21. Eraso, J. M. et al. The highly conserved MraZ protein is a transcriptional regulator in Escherichia coli. J. Bacteriol. 196, 2053-66 (2014).

22. Norioka, S., Ramakrishnan, G., Ikenaka, K. \& Inouye, M. Interaction of a transcriptional activator, OmpR, with reciprocally osmoregulated genes, ompF and ompC, of Escherichia coli. J. Biol. Chem. (1986).

23. Battesti, A., Majdalani, N. \& Gottesman, S. The RpoS-Mediated General Stress Response in Escherichia coli. Annu. Rev. Microbiol. 65, 189-213 (2011).

24. Al Mamun, A. A. M. et al. Identity and function of a large gene network underlying mutagenic repair of DNA breaks. Science (80-.), https://doi.org/10.1126/science.1226683, (2012).

25. Salmon, K. A. et al. Global gene expression profiling in Escherichia coli K12: Effects of oxygen availability and ArcA. J. Biol. Chem. 280, 15084-96 (2005).

26. Partridge, J. D. et al. Transition of Escherichia coli from aerobic to micro-aerobic conditions involves fast and slow reacting regulatory components. J. Biol. Chem. 282, 11230-7 (2007).

27. Nizam, S. A., Zhu, J., Ho, P. Y. \& Shimizu, K. Effects of arcA and arcB genes knockout on the metabolism in Escherichia coli under aerobic condition. Biochem. Eng. J. 44, 240-250 (2009).

28. Lobritz, M. A. et al. Antibiotic efficacy is linked to bacterial cellular respiration. Proc. Natl. Acad. Sci. USA 112, 8173-80 (2015).

29. Dwyer, D. J., Collins, J. J. \& Walker, G. C. Unraveling the physiological complexities of antibiotic lethality. Annu. Rev. Pharmacol. Toxicol. 55, 313-32 (2015).

30. Yamada, M. \& Saier, M. H. Positive and negative regulators for glucitol (gut) operon expression in Escherichia coli. J. Mol. Biol. 203, 569-83 (1988).

31. Herzberg, M., Kaye, I. K., Peti, W. \& Wood, T. K. YdgG (TqsA) controls biofilm formation in Escherichia coli K-12 through autoinducer 2 transport. J. Bacteriol. 188, 587-98 (2006).

32. Gama-Castro, S. et al. RegulonDB version 9.0: High-level integration of gene regulation, coexpression, motif clustering and beyond. Nucleic Acids Res. 44, D133-43 (2016).

33. Nyerges, Á. et al. A highly precise and portable genome engineering method allows comparison of mutational effects across bacterial species. Proc. Natl. Acad. Sci. USA 113, 2502-2507 (2016).

34. Bonde, M. T. et al. MODEST: A web-based design tool for oligonucleotide-mediated genome engineering and recombineering. Nucleic Acids Res. 42, (2014).

\section{Acknowledgements}

This work was supported, in part, by a Grant-in-Aid for Young Scientists (B) [17K15052], a Grant-in-Aid for Scientific Research (B) [17H03622], a Grant-inAid for Scientific Research (C) [19K06630], and a Grant-in-Aid for Scientific Research (S) [15H05746] from the JSPS; a Grant-in-Aid for Scientific Research on Innovative Areas 
[17H06389, 18H04807] from MEXT; and Incentive Research Projects from RIKEN. We thanks to Ms. Natsue Sakata, Ms. Aki Sakai, and Ms. Kumi Tanabe for technical assistance. We thank to the National BioResource Project (NIG, Japan): E. coli for providing the E. coli BW25113 and Keio collection strains.

\section{Author contributions}

T.H. and T.M. performed experimental evolution. T.H. and H.K. performed the construction of mutants. T.H. and C.F. analyzed the data. T.H. and C.F. designed the experiments and drafted the manuscript. C.F. managed overall the project.

\section{Competing interests}

The authors declare no competing interests.

\section{Additional information}

Supplementary information is available for this paper at https://doi.org/10.1038/s41598-020-60663-6.

Correspondence and requests for materials should be addressed to C.F.

Reprints and permissions information is available at www.nature.com/reprints.

Publisher's note Springer Nature remains neutral with regard to jurisdictional claims in published maps and institutional affiliations.

(c) (i) Open Access This article is licensed under a Creative Commons Attribution 4.0 International License, which permits use, sharing, adaptation, distribution and reproduction in any medium or format, as long as you give appropriate credit to the original author(s) and the source, provide a link to the Creative Commons license, and indicate if changes were made. The images or other third party material in this article are included in the article's Creative Commons license, unless indicated otherwise in a credit line to the material. If material is not included in the article's Creative Commons license and your intended use is not permitted by statutory regulation or exceeds the permitted use, you will need to obtain permission directly from the copyright holder. To view a copy of this license, visit http://creativecommons.org/licenses/by/4.0/.

(C) The Author(s) 2020 